\title{
Change in skin properties over the first 10 years of life: a cross-sectional study
}

\author{
Fanqi Kong $^{1,3} \cdot$ Carlos Galzote $^{2} \cdot$ Yuanyuan Duan $^{1}$
}

Received: 12 January 2017 / Revised: 28 June 2017 / Accepted: 11 July 2017 / Published online: 19 July 2017

(C) The Author(s) 2017. This article is an open access publication

\begin{abstract}
This study investigated skin characteristics in healthy Chinese children aged from 1 to 10 years and compared these findings with similar measures from the child's mothers. Children aged 1, 2, 3, 4, 5, and 10 years ( $n=29-30$ per age group) and the child's mothers were enrolled in a single-visit cross-sectional study. Clinical parameters evaluated on the face, ventral forearm, and calf were softness, smoothness, erythema, edema, rash, dryness, and scaling. Instrumental evaluations included transepidermal water loss, moisture content, and water-holding capacity. The clinical evaluations indicated a general decrease in softness, smoothness, and overall skin condition with increased child age. In general, the child's clinical scores were better than in adults. Children had a more permeable skin barrier that matured to adult values by approximately 5 years of age. Mothers had greater skin moisture than children. Clinical and instrumental measures were consistent with skin being softer and smoother and in better overall condition in younger children. As the skin matured with age, higher scores were observed. Instrumental measures demonstrated a more permeable skin barrier in younger children compared with older children and with adults.
\end{abstract}

Keywords Skin barrier - Skin condition - Transepidermal water loss $\cdot$ Water handling $\cdot$ Children $\cdot$ Adult

Fanqi Kong

fkong@its.jnj.com

Johnson \& Johnson AP Skin Testing Center, Shanghai, China

2 Johnson \& Johnson International (Singapore) Pte. Ltd, 2 International Business Park, Singapore 609930, Singapore

3 AP Clinical Operations, Johnson \& Johnson China Ltd., 3285 Dongchuan Road, Minhang, Shanghai, China

\section{Introduction}

The skin of infants and children is often characterized as smoother and softer than in the adult [8]. In infants, the stratum corneum is thinner, water handling is different, and natural moisturizing factor and skin lipid production are reduced compared with adults [4-6, 11]. Because of these developmental differences, the skin in children may be more sensitive to irritation and inflammation [7]. These differences can help explain some of the increased sensitivity of the infant to specific skin insults that are not as prevalent in adults and that can result in diaper dermatitis and atopic dermatitis [2].

The goals of this study were to investigate the changes in skin and skin barrier function in children aged from 1 to 10 years and to compare these findings with similar measures (clinical dermatologic characteristics as well as instrumental and physical characteristics) from the child's mothers.

\section{Methods}

\section{Study design}

This was a single-visit cross-sectional study in children of different ages (aged 1-5 and 10 years) performed from December 2012 to January 2013 in Shanghai, China. The mother of each child signed an informed consent form. The study was approved by an ethics committee or institutional review board and conformed to the latest revision of the Declaration of Helsinki and good clinical practice. Subjects were ethically compensated immediately after successful completion of the study in the form of small tokens, food, and/or a transportation allowance. 
Assessments were made in six groups of healthy children aged 1, 2, 3, 4, 5, and 10 years and in each child's mother. Skin properties as described below were assessed on the face, ventral forearm, and/or calf.

Inclusion and exclusion criteria are described in Table 1. The study subjects (mothers, children) were instructed to wash only with water $\geq 12 \mathrm{~h}$ before the clinic visit; no skin care product was to be used for $24 \mathrm{~h}$ prior to the visit.

\section{Dermatological assessments}

A single dermatologist evaluator performed all grading for all of the subjects in the study. The evaluated clinical parameters were overall skin condition, softness, smoothness, erythema (irritation), edema (irritation), rash (irritation), dryness, and scaling. Clinical assessments were made on the face, ventral forearm, and/or calf, and graded on a scale of 0 (none), 1-2 (very good), 3-4 (slight), 5-6 (medium or moderate), 7-8 (medium-severe), to 9 (severe). As described in Table 1, subjects with clinical scores in the moderate/medium-to-severe range were excluded from study entry.

\section{Instrumental evaluations}

Transepidermal water loss (TEWL) measurements on the cheek were obtained using a VapoMeter (Delfin Technologies, Finland), which uses a closed-chamber measurement principle to evaluate water evaporation from the skin [1].
The probe was placed into contact with the skin surface, and one measurement was recorded.

Skin moisture content was measured using a Corneometer $^{\circledR}(\mathrm{CM} 825$, Courage + Khazaka electronic $\mathrm{GmbH}$, Cologne, Germany) on the cheek and forearm (capacitance measure of surface skin moisture content, average of three readings per site) and a Skicon device (IBS Co. Hamamatsu, Japan), for high-frequency electric conductance measure of skin moisture content on the ventral forearm [9].

Water-holding capacity was tested using the Skicon device (IBS Co.) on the ventral forearm, as previously described [10]. After a baseline measurement was made, a drop of water was placed on the skin for $10 \mathrm{~s}$ and blotted. A measurement was then performed immediately thereafter $(0 \mathrm{~s})$ and at 30,60, and $120 \mathrm{~s}$. The entire test was repeated five times, with the average of three determinations being used to calculate water sorption and desorption (after removal of the minimum and maximum data points), according to the following formulas:

Water sorption $(\mathrm{WS})=\left(G_{0}-G_{\mathrm{BL}}\right) / 30$,

and

Water desorption $(\mathrm{WD})=\left(G_{30}-G_{0}\right) / 30$.

\section{Data analysis}

Dermatological assessments and instrumental assessments were summarized by mean \pm standard deviation

Table 1 Inclusion and exclusion criteria

\begin{tabular}{|c|c|}
\hline Inclusion criteria & Exclusion criteria \\
\hline $\begin{array}{l}\text { The participating mother is the one who regularly cares for the child. } \\
\text { The mother must } \geq 18 \text { years and be willing and able to follow all } \\
\text { study directions, accept skin examination of the study }\end{array}$ & $\begin{array}{l}\text { Child/mother participant who exhibits or is known to have atopic } \\
\text { dermatitis, eczema, or other inflammatory disease, or mild-to-severe } \\
\text { diaper rash or skin diseases/conditions that in the opinion of the inves- } \\
\text { tigator may affect the evaluation of study product or place the child at } \\
\text { undue risk }\end{array}$ \\
\hline $\begin{array}{l}\text { Participating mother must be willing to not bathe her child and herself } \\
\text { at least } 12 \mathrm{~h} \text { prior to the clinic visit and to have clean skin at the } \\
\text { clinic visit }\end{array}$ & $\begin{array}{l}\text { Any children and mothers with clinically determined moderate-to- } \\
\text { severe dryness or clinically determined erythema, rash, or other skin } \\
\text { condition }\end{array}$ \\
\hline \multirow[t]{6}{*}{$\begin{array}{l}\text { Mother must be willing to not apply any skin care product to the child } \\
\text { and herself for } 24 \mathrm{~h} \text { before visit until after the visit is completed }\end{array}$} & $\begin{array}{l}\text { Any condition requiring use of a topical or oral OTC or prescription } \\
\text { medication, which, in the investigator's judgment, makes the subject } \\
\text { ineligible or places the subject at risk. Child vitamins were allowed }\end{array}$ \\
\hline & Individuals currently involved in another clinical study of any type \\
\hline & $\begin{array}{l}\text { Child participants who experienced moderate-to-severe diaper rash } \\
\text { within } 1 \text { week prior to study start }\end{array}$ \\
\hline & $\begin{array}{l}\text { Child/mother participant who has experienced unusual hypersensitivity } \\
\text { or allergic reactivity to fragrances and/or reaction/irritation to skin } \\
\text { care toiletry products }\end{array}$ \\
\hline & $\begin{array}{l}\text { Child/mother participant with a known condition of asthma or any } \\
\text { related breathing problems and/or for whom there is a family history } \\
\text { of asthma }\end{array}$ \\
\hline & $\begin{array}{l}\text { Mother of the child is an employee of the investigator or study center or } \\
\text { is a family member of the employees or the investigator }\end{array}$ \\
\hline
\end{tabular}


(SD), unless otherwise specified. The relationship of the skin evaluation data between babies and their mother was analyzed using Pearson correlation coefficient. The crosssectional evaluation pertains to group comparisons of skin evaluation data of both babies and their mothers. It included comparisons of all age groups and pairwise comparisons among the age groups. Univariate cross-sectional comparisons (testing the difference among the age groups) were conducted using analysis of variance (ANOVA) and were assessed by non-parametric Kruskal-Wallis test. Differences between means were evaluated by the non-parametric Mann-Whitney $U$ test or by the independent $t$ test. The significance level for statistical tests of differences among the groups was set at 0.05 . The Bonferroni method was used to account for cases of multiple comparisons.

\section{Results}

In this study, 384 eligible subjects were recruited (192 children and 192 mothers) and 358 subjects completed the study. Demographics are shown in Table 2. There was an even distribution of boys and girls in the study and 30 children in each age group (except for 3 years with $n=29$ ). The average age of mothers ranged from $29 \pm 4$ years for 1 year to $36 \pm 7$ years for 10 years.

\section{Dermatological assessments}

\section{Assessments in children}

In the assessments of the children's facial skin, significant differences across age groups $(p<0.05)$ were observed for all dermatology variables (Fig. 1), except edema (not shown) when analyzed by ANOVA and Kruskal-Wallis test. In the pairwise age-group comparisons, there were generally no significant differences between age groups in mean scores for facial softness, smoothness, overall skin condition, and erythema, edema, or rash (measures of irritation) (Fig. 2). Differences among age groups were noted for dryness and scaling, possibly because dryness and scaling scores of 3- and 5 years were higher than in the other groups (Fig. 1). In spite of the fact that mean values for facial dryness and scaling in 3 and 5 years were higher than in the other groups, these values were all less than 2 , which was still considered in the very good range.

In the assessments of the ventral forearm and calf, there were significant differences across groups for softness, smoothness, overall skin condition, dryness, and scaling $(p<0.05)$, but not for erythema, edema, and rash $(p>0.05)$. Pairwise age-group comparisons demonstrated differences between age groups in softness, smoothness, overall skin condition, dryness, and scaling; again, differences in dryness and scaling were likely to be driven by the slightly higher scores in the 3-and 5-year-old groups (Fig. 1). Overall, the clinical data from the assessments of the children's skin indicated a general increase in score with age in softness, smoothness, and overall skin condition.

\section{Instrumental evaluations}

TEWL measured on the cheek was greater in 1-year-old children when compared with children older than age 5 or adults (Fig. 2a).This finding was consistent with a more permeable skin barrier in the younger children.

Skin surface moisture, as measured by capacitance (Corneometer), was generally not influenced by children's age on the cheek; however, on the ventral forearm, capacitance at age 10 was reduced compared with ages 1 and 2 (Fig. 2b). Corneometer measurement of skin moisture was significantly greater in mothers than in children in all age groups on the cheek, and it was greater on the ventral forearm at all ages older than 1 (Fig. 2b).

Skin moisture, as measured by high-frequency conductance (Skicon) on the ventral forearm, decreased significantly and differed between age groups in children, from a mean value of 78 in 1 year to 35 in 10 years (ANOVA, $p<0.001$; Fig. 2c). Pairwise comparisons showed significantly higher conductance in 1 year versus 3-, 4-, and 10 years, suggesting a trend for a decrease in baseline skin conductance at this site with age. This decrease in forearm skin moisture was consistent with Corneometer measurements at the same site. No consistent difference between mothers and children was observed in forearm conductance; however, mothers had greater conductance than 4and 10 years.

Table 2 Participant demographics by children's age

\begin{tabular}{lllllll}
\hline & 1 year $(n=30)$ & 2 years $(n=30)$ & 3 years $(n=29)$ & 4 years $(n=30)$ & 5 years $(n=30)$ & 10 years $(n=30)$ \\
\hline Males, $n(\%)$ & $16(53 \%)$ & $13(43 \%)$ & $15(52 \%)$ & $15(50 \%)$ & $16(53 \%)$ & $15(50 \%)$ \\
Females, $n(\%)$ & $14(47 \%)$ & $17(57 \%)$ & $14(48 \%)$ & $15(50 \%)$ & $14(47 \%)$ & $15(50 \%)$ \\
$\begin{array}{l}\text { Mother's age, years } \\
(\text { mean } \pm \text { SD) }\end{array}$ & $29 \pm 4$ & $29 \pm 4$ & $32 \pm 9$ & $32 \pm 7$ & $33 \pm 6$ & $36 \pm 7$ \\
\hline
\end{tabular}

$S D$ standard deviation 

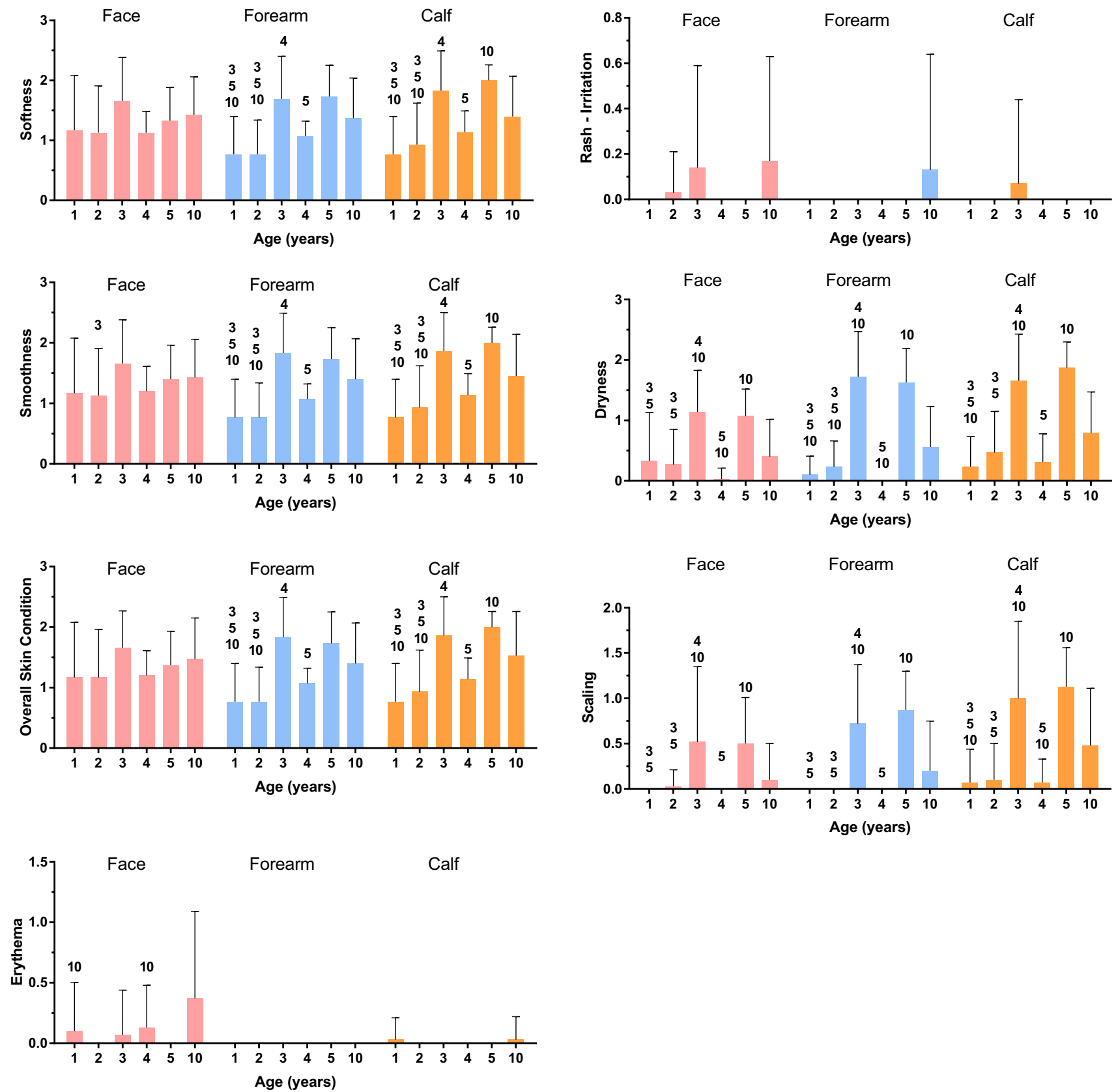

Fig. 1 Dermatological assessments. A number above the bar in an age group indicate that a significant difference was between this age group and the other age groups identified

Water-holding capacity was monitored by measuring the uptake (sorption) and release (desorption) of water from the skin. Water sorption and desorption profiles were obtained from children and their mothers on the ventral forearm immediately after baseline using high-frequency conductance. Values in 1 year and their mothers at baseline and at 0, 30, 60, and $120 \mathrm{~s}$ are shown in Fig. 2d, where it was found that there was a higher water-holding trend in children than adults. Desorption values in 2 years were lower compared with those aged 3 and 10 years (Fig. 2e), indicating greater tendency of the skin to hold water.

Overall skin hydration can be considered as a ratio of skin moisture (water content) to water loss. The ratio of water content (as measured by Corneometer) to TEWL (as measured by Vapometer) suggested that overall skin hydration in children increased through age 5 , at which point it began to approach the adult value (i.e., the ratio observed in mothers; Fig. 3). 
a

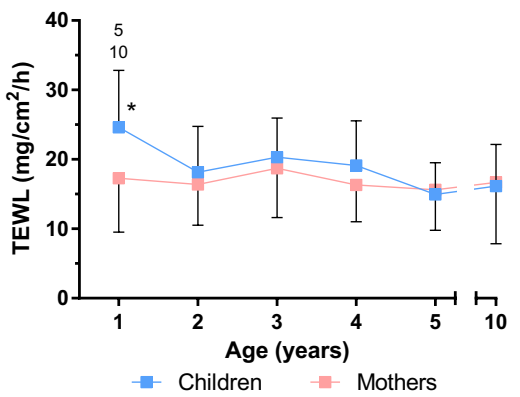

C

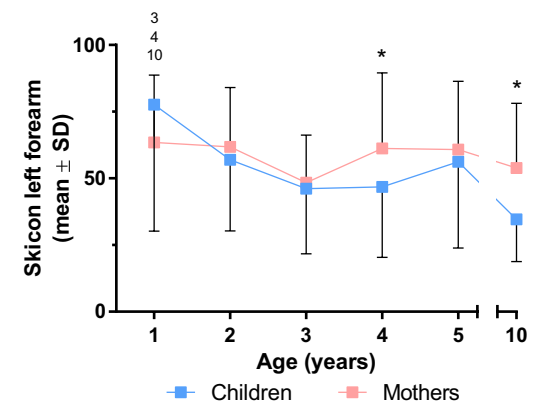

b

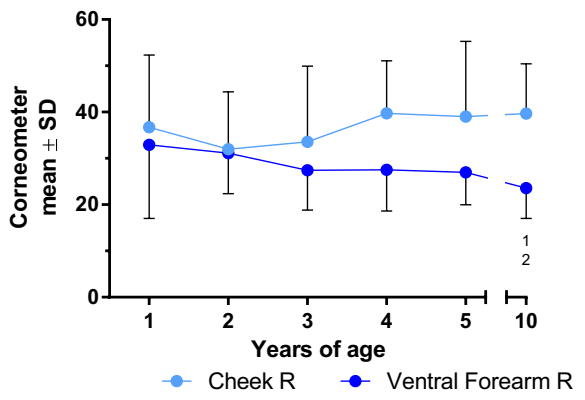

d

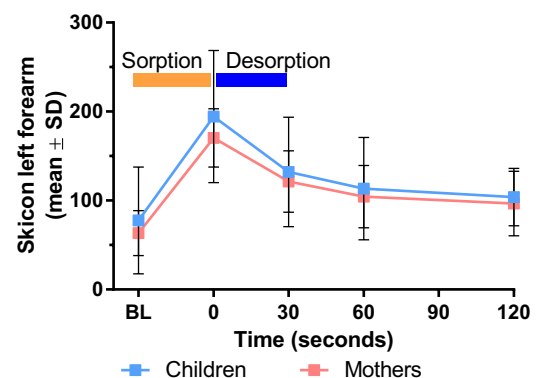

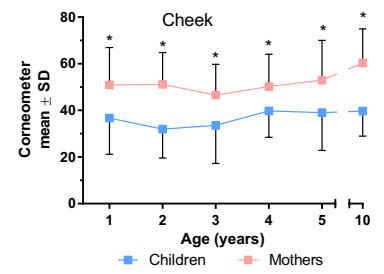

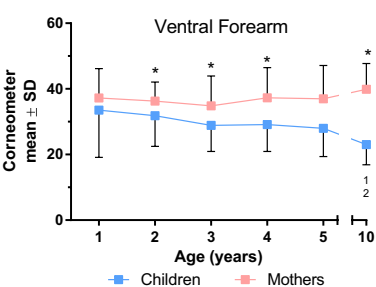

Children (years) Mothers

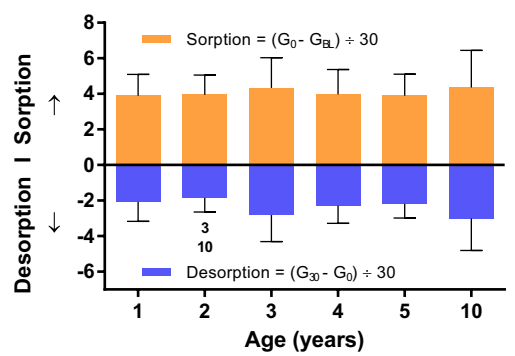

Fig. 2 Measures of skin barrier function and skin moisture content or hydration a TEWL, b Corneometer (capacitance) in cheek and ventral forearm, c Skicon (conductance), d water-handling time course in 1 year, and e water-handling changes with age. A number above the bar or symbol in a children's age group mean that a significant difference was observed between this age group (the bar) and the groups shown $(p<0.05) .{ }^{*} p<0.05$ children versus mothers $(t$ test $)$

instrumentally) of Chinese children. The clinical evaluations indicated a general decrease in softness, smoothness, and overall skin condition (measured as increased scores) with children's age. Although our study was performed in a homogeneous ethnic group, our findings were consistent with those in several different ethnic groups [4]. As children age, there are significant decreases in TEWL and a concomitant decrease in moisture content, plateauing at about 5 years of age [11]. The scores in children versus adults seen in this study are consistent with a maturing of the skin barrier over time [4, 11].

Unexpectedly, the 3 years had the highest scores for dermatology assessments. The reason for the differences is not clear, and could not be explained by a difference where the child was born (Shanghai versus other locations). The mean scores in this group were still within the very good range $(<2)$, meaning that although slightly higher, they were not considered out of the ordinary or abnormal. Individuals were not excluded from study entry unless the characteristics were clinically determined to be moderate to severe (scores $>7$ ).
This study demonstrated significant changes with age in skin characteristics (measured clinically and 
The water sorption-desorption test can be used to provide information about the water-holding capacity of the skin over a short time scale [10]. One-year-old children had slightly greater sorption than their mothers in this study. This finding is consistent with a more permeable skin barrier to water in younger children than in adults $[4,5,11]$, although the difference has not been observed by all investigators [3].

Two different measures (Corneometer, Skicon) were used to evaluate possible changes in skin moisture content with age on the ventral forearm. Both methods showed similar results, reinforcing the concept that moisture content at this site was highest in 1 year and decreased with age (however, on the cheek, no consistent change was observed by age). The finding that the children's mothers had greater skin moisture content than the children contrasted with findings from others [4] who demonstrated moisture content of adults was lower than infants/toddlers. These differences may perhaps have been due to previous moisturizer use by the mothers or to differences in technique and/or site of measurement. Overall hydration, measured as the ratio of Corneometer value to TEWL [12], increased with age, and suggested that the maturation of the skin barrier (e.g., reduced TEWL) had a greater effect on skin moisture than the smaller decrease in Corneometer or Skicon value and that the overall effect was to increase skin hydration.

This was a cross-sectional study performed over a short period of time and not a longitudinal study. We cannot assume that changes observed in one of the studied age groups will progress as these specific children age. In addition, each group of subjects was relatively small ( $\leq 30$ individuals) making generalizations to other populations difficult. Because the age cohorts were small, we did not make any attempt to analyze the data stratified by sex and, therefore, were unable to determine whether sex influenced skin properties subjects studied.

In summary, this study used both clinical and instrumental methods to assess skin condition and skin barrier function in children and adults. Clinically, skin was generally perceived to be softer and smoother and in better overall condition in younger children. As expected with skin maturation, the clinical scores tended to increase with age.

Acknowledgements The authors wish to thank the families of the study subjects for participating in this study. This study was fully funded by Johnson \& Johnson International Pte. Ltd (Singapore) and all authors are employees of Johnson \& Johnson. Medical writing and editorial support was provided by Alex Loeb, PhD, CMPP of Evidence Scientific Solutions (Philadelphia, PA, USA) and was funded by Johnson \& Johnson Consumer Inc. (Skillman, NJ, USA).

\section{Compliance with ethical standards}

Ethical approval All procedures performed in studies involving human participants were in accordance with the ethical standards of the institutional and/or national research committee and with the 1964 Helsinki declaration and its later amendments or comparable ethical standards.

Open Access This article is distributed under the terms of the Creative Commons Attribution 4.0 International License (http:// creativecommons.org/licenses/by/4.0/), which permits unrestricted use, distribution, and reproduction in any medium, provided you give appropriate credit to the original author(s) and the source, provide a link to the Creative Commons license, and indicate if changes were made.

\section{References}

1. De Paepe K, Houben E, Adam R, Wiesemann F, Rogiers V (2005) Validation of the VapoMeter, a closed unventilated chamber system to assess transepidermal water loss vs. the open chamber Tewameter ${ }^{\circledR}$. Skin Res Technol 11:61-69. doi:10.1111/j.1600-0846.2005.00101.x

2. Del Rosso JQ, Levin J (2011) The clinical relevance of maintaining the functional integrity of the stratum corneum in both healthy and disease-affected skin. J Clin Aesthet Dermatol 4:22-42

3. Fluhr JW, Pfisterer S, Gloor M (2000) Direct comparison of skin physiology in children and adults with bioengineering methods. Pediatr Dermatol 17:436-439

4. Mack MC, Chu MR, Tierney NK, Ruvolo E Jr, Stamatas GN, Kollias N, Bhagat K, Ma L, Martin KM (2016) Water-holding and transport properties of skin stratum corneum of infants and toddlers are different from those of adults: studies in three geographical regions and four ethnic groups. Pediatr Dermatol 33:275-282. doi:10.1111/pde. 12798

5. Nikolovski J, Stamatas GN, Kollias N, Wiegand BC (2008) Barrier function and water-holding and transport properties of infant stratum corneum are different from adult and continue to develop through the first year of life. J Investig Dermatol 128:1728-1736. doi:10.1038/sj.jid.5701239

6. Piérard GE, Piérard-Franchimont C, Lê T, Lapière C (1987) Patterns of follicular sebum excretion rate during lifetime. Arch Dermatol Res 279(Suppl):S104-S107

7. Stamatas GN, Morello AP, Mays DA (2013) Early inflammatory processes in the skin. Curr Mol Med 13:1250-1269

8. Stamatas GN, Nikolovski J, Luedtke MA, Kollias N, Wiegand BC (2010) Infant skin microstructure assessed in vivo differs from adult skin in organization and at the cellular level. Pediatr Dermatol 27:125-131. doi:10.1111/j.1525-1470.2009.00973.x

9. Tagami H (1994) Quantitative measurements of water concentration of the stratum corneum in vivo by high-frequency current. Acta Derm Venereol Suppl (Stockh) 185:29-33

10. Tagami H, Kanamaru Y, Inoue K, Suehisa S, Inoue F, Iwatsuki K, Yoshikuni K, Yamada M (1982) Water sorption-desorption test of the skin in vivo for functional assessment of the stratum corneum. J Investig Dermatol 78:425-428

11. Walters RM, Khanna P, Chu M, Mack MC (2016) Developmental changes in skin barrier and structure during the first 5 years of life. Skin Pharmacol Physiol 29:111-118. doi:10.1159/000444805

12. Yuan C, X-m Wang, Y-m Tan (2011) Short-term repairing function of moisture cream on lactic acid stingers. Chin J Aesthet Med 20:1121-1123 